\section{(2) OPEN ACCESS}

\title{
Characterisation of patients with severe asthma in the UK Severe Asthma Registry in the biologic era
}

\author{
David J Jackson, ${ }^{1,2}$ John Busby, ${ }^{3}$ Paul E Pfeffer (D) , ${ }^{4}$ Andrew Menzies-Gow, ${ }^{5}$ \\ Thomas Brown, ${ }^{6}$ Robin Gore, ${ }^{7}$ Martin Doherty, ${ }^{8}$ Adel H Mansur (i) , 9,10 \\ Simon Message, ${ }^{11}$ Robert Niven, ${ }^{12}$ Mitesh Patel, ${ }^{13}$ Liam G Heaney, ${ }^{14,15}$ on behalf of the \\ UK Severe Asthma Registry
}

\begin{abstract}
- Supplement material is published online only. To view, please visit the journal online (http://dx.doi.org/10.1136/ thoraxjnl-2020-215168).
\end{abstract}

For numbered affiliations see end of article.

\section{Correspondence to} Professor Liam G Heaney, Centre for Experimental Medicine, Queen's University Belfast, Belfast, Belfast, UK; l.heaney@qub.ac.uk

Received 29 April 2020 Revised 15 September 2020 Accepted 14 October 2020 Published Online First 9 December 2020

\section{Check for updates}

(C) Author(s) (or their employer(s)) 2021. Re-use permitted under CC BY-NC. No commercial re-use. See rights and permissions. Published by BMJ.

To cite: Jackson DJ, Busby J, Pfeffer PE, et al. Thorax 2021;76:220-227.

\begin{abstract}
Background The UK Severe Asthma Registry (UKSAR) is the world's largest national severe asthma registry collecting standardised data on referrals to UK specialist services. Novel biologic therapies have transformed the management of type 2(T2)-high severe asthma but have highlighted unmet need in patients with persisting symptoms despite suppression of T2-cytokine pathways with corticosteroids.
\end{abstract}

Methods Demographic, clinical and treatments characteristics for patients meeting European Respiratory Society / American Thoracic Society severe asthma criteria were examined for 2225 patients attending 15 specialist severe asthma centres. We assessed differences in biomarker low patients (fractional exhaled nitric oxide (FeNO) $<25 \mathrm{ppb}$, blood eosinophils $<150 / \mu \mathrm{L}$ ) compared with a biomarker high population (FeNO $\geq 25 \mathrm{ppb}$, blood eosinophils $\geq 150 / \mu \mathrm{L}$ ).

Results Age (mean 49.6 (14.3) y), age of asthma onset (24.2 (19.1) y) and female predominance (62.4\%) were consistent with prior severe asthma cohorts. Poor symptom control (Asthma Control Questionnaire-6: 2.9 (1.4)) with high exacerbation rate (4 (IQR: 2, 7)) were common despite high-dose treatment (51.7\% on maintenance oral corticosteroids (mOCS)). $68.9 \%$ were prescribed biologic therapies including mepolizumab $(50.3 \%)$, benralizumab $(26.1 \%)$ and omalizumab (22.6\%). T2-low patients had higher body mass index (32.1 vs $30.2, p<0.001$ ), depression/anxiety prevalence $(12.3 \%$ vs $7.6 \%, p=0.04)$ and $m 0 C S$ use $(57.9 \%$ vs $42.1 \%, p<0.001)$. Many T2-low asthmatics had evidence of a historically elevated blood eosinophil count $(0.35$ $(0.13,0.60))$.

Conclusions The UKSAR describes the characteristics of a large cohort of asthmatics referred to UK specialist severe asthma services. It offers the prospect of providing novel insights across a range of research areas and highlights substantial unmet need with poor asthma control, impaired lung function and high exacerbation rates. T2-high phenotypes predominate with significant differences apparent from T2-low patients. However, T2-low patients frequently have prior blood eosinophilia consistent with possible excessive corticosteroid exposure.

\section{INTRODUCTION}

Severe asthma has been defined by the European Respiratory Society / American Thoracic Society

\section{Key messages}

\section{What is the key question?}

What are the demographic, clinical and treatment characteristics of patients referred to UK specialist severe asthma services?

\section{What is the bottom line?}

Patients have substantial unmet need despite significant background treatment. Clear differences exist between T2-low and T2-high phenotypes although the majority of the T2-low group have evidence of prior T2-high disease, possibly reflecting effective suppression of $\mathrm{T} 2$ pathways with corticosteroids.

\section{Why read on?}

This study reports a comprehensive description of severe asthma patients in the UK from the largest national registry of its kind in the world.

(ERS/ATS) as asthma which requires treatment with high-dose inhaled corticosteroids (ICS) plus a second controller (and/or systemic corticosteroids) to prevent it from becoming 'uncontrolled' or which remains 'uncontrolled' despite this therapy. ${ }^{1}$ Although some estimates have suggested that severe asthma affects approximately $5 \%-10 \%$ of the total asthma population, ${ }^{12}$ it is well recognised that many patients prescribed high-dose inhaled treatment remain poorly controlled because of suboptimal adherence to treatment or poor inhaler technique despite regular use. Once these two critical aspects of asthma care have been addressed the true prevalence of patients who remain poorly controlled on high-dose inhaled treatment falls to approximately $3 \%-4 \% .^{3}$ Exploring the clinical characteristics and disease expression in a large well-characterised severe asthma cohort is vital to understanding the heterogeneity and unmet need in these patients.

The British Thoracic Society (BTS) Difficult Asthma Registry was established in 2007 and was the first national registry to describe the clinical and demographic characteristics in a large patient group with well-characterised severe asthma. ${ }^{4}$ This 'real-world' data describing a population frequently underrepresented in clinical trials due to comorbidities or smoking history has been useful in helping to define clinical outcomes and health economic 
costs associated with routine clinical care, as well as the significant morbidity associated with systemic corticosteroids in this population..$^{5-14}$

With our improved understanding of the central role of type-2 (T2) inflammation in severe asthma alongside the advent of biologic therapies targeting elements of the T2 inflammatory cascade, the original BTS Registry underwent a number of important structural changes and was renamed the UK Severe Asthma Registry (UKSAR). The data to be collected were initially agreed by consensus between UK Expert Physicians and in a subsequent Delphi Consensus of International Severe Asthma Experts, there was 95\% agreement with the UKSAR data fields. ${ }^{15}$ The patient population is well-characterised, with the participating centres all being specialist multidisciplinary units for asthma care with all patients undergoing systematic assessment prior to inclusion in the registry.

The aims of the current article are to describe the demographic and clinical characteristics of severe asthma patients in the UKSAR, to describe current biologic selection in eligible patients and to examine the difference between patients who are T2 biomarker-high and T2 biomarker-low at registration.

\section{MATERIALS AND METHODS}

Patients in UKSAR are enrolled to the registry after referral to Specialist UK Severe Asthma Centres with uncontrolled asthma (ie, severe symptoms or frequent exacerbations) at the Global Initiative for Asthma (GINA) treatment steps 4 and 5. The UKSAR has database ethical approval from the Office of Research Ethics Northern Ireland (15/NI/0196) and all patients provide written informed consent. All clinical centres in England are part of the NHS England Specialist Commissioning Network and operate on a 'hub and spoke' basis which aims to provide standardised multidisciplinary assessment and access to therapies under guidance issued by the National Institute for Clinical and Healthcare Excellence. Northern Ireland has a single-centre regional service (Belfast) and UKSAR also includes a single specialist regional centre in Scotland (online supplemental appendix 1).

Data were collected from November 2016 to February 2020. Baseline (at the time of registration) demographic and clinical variables are listed in (online supplemental appendix 2, but in brief, UKSAR captures 105 core variables classified into nine categories: patient demographics, medical history, investigations, lung function, allergy testing, Asthma Control Questionnaire, EuroQoL Questionnaire, asthma medication, and systematic assessment summary and management plan. Follow-up data are collected annually. The information recorded at each visit are listed in online supplemental appendix 3, however, follow-up data are not reported in this manuscript. The number of exacerbations was defined as a count of exacerbations requiring rescue systemic corticosteroids in the past 12 months. The number of hospitalisations and emergency department admissions for asthma was the number in the past 12 months. Asthma control was measured using the Asthma Control Questionnaire-6 (ACQ6). In this analysis, all patients were assessed by their treating clinician as fulfilling the criteria for severe asthma as defined by ERS/ATS Guidelines. ${ }^{1}$ T2-biomarker low patients were defined as fractional exhaled nitric oxide (FeNO) $<25 \mathrm{ppb}$ and blood eosinophil count $<150$ cells $/ \mu \mathrm{L}$ at registration. These cut-points were chosen as phase 3 clinical trials of targeted antiinterleukin 5 (IL5) and anti-IL4R biologic therapies have shown little benefit below these thresholds in patients with severe asthma. ${ }^{16} 17$ The T2-high comparator cohort was defined when both these biomarkers were above these thresholds.
All patients entered into UKSAR have undergone a thorough systematic multidisciplinary assessment as previously described in UK services. ${ }^{18} 19$ At this assessment, centres are asked to confirm that patients fulfil the ERS/ATS criteria for asthma that they have evaluated and optimised adherence to their current treatment plan and a management plan including additional treatments is registered (see online supplemental). Consecutive patients completing this assessment, and consenting to registry participation are entered on to the UKSAR.

\section{Statistical analysis}

This was a hypothesis generating study with no pre-specified hypotheses. Descriptive statistics were calculated for the entire cohort and for specific patient subgroups. Mean (with SD) and median (with IQR) were presented for continuous variables as appropriate. Categorical variables were summarised using counts and percentages. Univariate hypothesis tests between groups were conducted using t-test, Mann-Whitney U test or $\chi^{2}$ test. All analyses were conducted using the STATA V.16 software package (StataCorp).

\section{RESULTS}

\section{Demographic, clinical characteristics and comorbidities}

Two thousand two hundred and twenty-five adult patients with severe asthma according to ERS/ATS criteria registered between November 2016 and February 2020 from 15 centres across the UK are included in this data. The mean age at registration was 49.6 years (14.3) with a mean age at onset of 24.2 years (19.1). $62.4 \%$ were female and $79.1 \%$ Caucasian. Self-reported atopic disease was recorded in $62.8 \%$; in those reporting a history of atopic disease and proceeding to skin prick test or radioallergosorbent (RAST) test, 72\% (858 of 1189 patients) were positive to a perennial environmental allergen. In those reporting no atopic disease, 29\% (207 of 707) were positive to skin prick or RAST-positive suggesting clinical history was of limited utility in identifying atopy in this group. Only 3.5\% reported current smoking whereas $68.3 \%$ of patients were never-smokers. Patients were poorly controlled with a mean ACQ6 of 2.9 (1.4), and a median of 4 (IQR: 2,7) exacerbations in the previous year. Lung function highlighted significant airflow obstruction and gas trapping with a $\mathrm{FEV}_{1}$ of 65.3 (21.3) \% predicted, a $\mathrm{FEV}_{1} / \mathrm{FVC}$ of $63.4 \%(17.3 \%)$ and a residual volume of $132 \%$ (44.1\%). Reported comorbidities included gastro-oesophageal reflux (16.9\%), nasal polyposis (16.4\%), depression or anxiety $(8.3 \%)$ and eczema (2.9\%) (table 1$)$.

\section{Biomarker profile}

The median blood eosinophil count at registration was 0.33 (IQR: $0.16,0.60)$ cells $\times 10^{9} / \mathrm{L}$ with the median highest historical result recorded as 0.62 (IQR: $0.40,1.00$ ) cells $\times 10^{9} / \mathrm{L}$. Median FeNO levels were 39.0 (IQR: 20.0, 75.0) ppb and the median total IgE was 181 (IQR: 60, 480) IU/mL (table 2). These levels highlight a predominantly $\mathrm{T} 2$-high profile in patients entered into the UKSAR.

\section{Asthma medication patterns}

All patients were on high-dose ICS and long-acting beta agonist therapy with a median ICS dose of 2000 (IQR: 1600, 2000) mcg BDP equivalent. Of these, 53.5\% were additionally on a longacting antimuscarinic antagonist (LAMA), 48.9\% on a leukotriene receptor antagonist (LTRA) and $27.2 \%$ on theophylline. There was no evidence of greater airflow obstruction among those prescribed a LAMA ( $\mathrm{FEV}_{1} / \mathrm{FVC}$ ratio: 63.2\%) compared 


\begin{tabular}{|c|c|}
\hline Number of patients & 2225 \\
\hline Age at first assessment & $49.6(14.3)$ \\
\hline $18-34$ & $378(17.0 \%)$ \\
\hline $35-54$ & $979(44.0 \%)$ \\
\hline $55-79$ & 855 (38.5\%) \\
\hline $80+$ & $11(0.5 \%)$ \\
\hline Age at onset of symptoms & $24.2(19.1)$ \\
\hline$<12$ & $715(36.2 \%)$ \\
\hline $12-18$ & $184(9.3 \%)$ \\
\hline$>18$ & $1076(54.5 \%)$ \\
\hline \multicolumn{2}{|l|}{ Gender } \\
\hline Female & $1389(62.4 \%)$ \\
\hline Male & $836(37.6 \%)$ \\
\hline \multicolumn{2}{|l|}{ Ethnicity } \\
\hline Caucasian & $1744(79.1 \%)$ \\
\hline Non-Caucasian & $462(20.9 \%)$ \\
\hline $\mathrm{BMI}\left(\mathrm{kg} / \mathrm{m}^{2}\right)$ & $30.8(7.1)$ \\
\hline \multicolumn{2}{|l|}{ Smoking status } \\
\hline Never smoker & $1490(68.3 \%)$ \\
\hline Ex-smoker & $617(28.3 \%)$ \\
\hline Current smoker & $76(3.5 \%)$ \\
\hline Atopic disease & $1378(62.8 \%)$ \\
\hline Spinal bone density (T-Score) & $-0.7(1.4)$ \\
\hline Femoral neck bone density (T-Score) & $-0.4(1.1)$ \\
\hline $\mathrm{FEV}_{1}(\%$ predicted $)$ & $65.3(21.3)$ \\
\hline FVC (\% predicted) & $83.1(19.7)$ \\
\hline $\mathrm{FEV}_{1} / \mathrm{FVC}$ & $63.4(17.3)$ \\
\hline Residual volume (\% predicted) & $132.0(44.1)$ \\
\hline Total lung capacity (\% predicted) & $103.9(18.7)$ \\
\hline ACQ6 Score & $2.9(1.4)$ \\
\hline Exacerbations requiring rescue steroids in last year & $4(2,7)$ \\
\hline Exacerbations requiring hospital admission in last year & $0(0,1)$ \\
\hline Invasive ventilation (ever) & $193(10.0 \%)$ \\
\hline Eczema & $63(2.9 \%)$ \\
\hline Nasal polyps & $356(16.4 \%)$ \\
\hline Gastro-oesophageal reflux & $367(16.9 \%)$ \\
\hline Depression or anxiety & $180(8.3 \%)$ \\
\hline
\end{tabular}

${ }^{*}$ Mean (SD), median (IQR) or count (\%) as appropriate.

$A C Q$, Asthma Control Questionnaire; BMI, body mass index.

with those not prescribed one $\left(\mathrm{FEV}_{1} / \mathrm{FVC}: 64.2 \%\right), \mathrm{p}=0.111$. $51.7 \%$ were on maintenance oral corticosteroids (mOCS) with a median daily dose of 10 (IQR: 5,15$) \mathrm{mg}$ prednisolone. $68.9 \%$ were prescribed biologic therapy including mepolizumab (50.3\%), omalizumab (22.6\%), benralizumab (26.1\%), reslizumab $(0.6 \%)$ and dupilumab (0.3\%) (table 2$)$.

\section{Comparison of biologic prescribed and non-prescribed patients with severe asthma}

A comparison of the demographic and clinical characteristics between patients prescribed biologic therapies and those treated with conventional therapies highlighted significant differences in several domains. While the age and gender distribution were

\begin{tabular}{lc}
\hline Table 2 & Medication and biomarkers* \\
\hline Number of patients & 2225 \\
Blood eosinophil count $\left(\mathrm{N} / 10^{9} \mathrm{~L}\right)$ & $0.33(0.16,0.60)$ \\
\hline Highest blood eosinophil count $\left(\mathrm{N} / 10^{9} \mathrm{~L}\right) \dagger$ & $0.62(0.40,1.00)$ \\
\hline FeNO $(\mathrm{ppb})$ & $39.0(20.0,75.0)$ \\
\hline IgE $(\mathrm{IU} / \mathrm{mL})$ & $181(60,480)$ \\
\hline Maintenance oral steroids & $1142(51.7 \%)$ \\
\hline Maintenance oral steroid dose $(\mathrm{mg})$ & $10(5,15)$ \\
\hline Inhaled steroid dose (mcg, BDP equivalent) & $2000(1600,2000)$ \\
\hline LAMA & $1161(53.5 \%)$ \\
\hline Theophylline & $595(27.2 \%)$ \\
\hline SABA & $2089(95.1 \%)$ \\
\hline Leukotriene receptor antagonist & $1048(48.9 \%)$ \\
\hline Maintenance macrolide & $199(9.3 \%)$ \\
\hline Nebuliser & $533(24.5 \%)$ \\
\hline Prior anti-lgE therapy & $251(11.5 \%)$ \\
\hline Initiate/continue biologic therapy & $1524(68.9 \%)$ \\
\hline Biologic therapy name & \\
\hline Omalizumab & $329(22.6 \%)$ \\
\hline Dupilumab & $5(0.3 \%)$ \\
\hline Mepolizumab & $731(50.3 \%)$ \\
\hline Benralizumab & $380(26.1 \%)$ \\
\hline Reslizumab & $9(0.6 \%)$ \\
\hline Mean (SD) & \\
\hline
\end{tabular}

${ }^{*}$ Mean (SD), median (IQR) or count (\%) as appropriate.

†Highest blood count is the highest recorded in available prior medical records. FeNO, fractional exhaled nitric oxide; LAMA, long-acting antimuscarinic antagonist; SABA, short acting $\beta 2$-agonist.

similar, a greater proportion of those prescribed biologics were Caucasian $(80.6 \%$ vs $75.5 \% ; \mathrm{p}=0.024)$. Patients on biologics were also more likely to be never-smokers $(70.8 \%$ vs $62.7 \%)$ and less likely to be current smokers $(2.2 \%$ vs $6.4 \% ; \mathrm{p}<0.001)$.

Patients treated with biologics had evidence of more severe airflow obstruction $\left(\mathrm{FEV}_{1} 64.2 \%\right.$ vs $\left.67.7 \% ; \mathrm{p}<0.001\right)$, greater air trapping (residual volume $136.3 \%$ vs $120.7 \%$; $p<0.001$ ), higher registration $(0.36$ vs $0.30 ; \mathrm{p}<0.001)$ and historic $(0.70$ vs $0.50 ; \mathrm{p}<0.001)$ blood eosinophil and FeNO levels $(41 \mathrm{ppb}$ vs 36 $\mathrm{ppb} ; \mathrm{p}<0.001)$. Prescription of non-biologic agents also differed significantly between patients additionally on biologic therapies. Specifically, patients starting on biologics had higher rates of mOCS $(59.9 \%$ vs $33.5 \% ; \mathrm{p}<0.001)$ and LAMA use $(54.6 \%$ vs $50.9 \% ; \mathrm{p}<0.001)$, despite lower LTRA use $(45.4 \%$ vs $56.2 \%$; $\mathrm{p}<0.001$ ) (table 3).

Further differences between biologic and non-biologic treated patients were observed with regards to comorbidities, with nasal polyposis being more prevalent in biologic patients $(18.2 \%$ vs $12.3 \% ; \mathrm{p}<0.001)$, while gastro-oesophageal reflux $(13.2 \%$ vs $25.4 \% ; \mathrm{p}<0.001)$ and psychological morbidity $(6.2 \%$ vs $13.0 \%$; $\mathrm{p}<0.001)$ being more common in biologic untreated patients (table 3).

\section{Clinical characteristics of anti-IgE versus anti-IL5/5R treated patients}

Analysis of the clinical and phenotypic characteristics of the biologic treated patients highlighted a number of significant differences between those prescribed the anti-IgE $\mathrm{mAb}$ omalizumab from those prescribed the anti-IL-5/5R mAbs mepolizumab, reslizumab or benralizumab. 
Table 3 Biologic population versus non-biologic population*

\begin{tabular}{|c|c|c|c|}
\hline & $\begin{array}{l}\text { No biologic therapy } \\
(n=687)\end{array}$ & $\begin{array}{l}\text { Biologic therapy } \\
(n=1524)\end{array}$ & $P$ value \\
\hline Age at first assessment & $49.1(14.3)$ & $49.7(14.3)$ & 0.332 \\
\hline $18-34$ & $118(17.2 \%)$ & $257(16.9 \%)$ & \\
\hline $35-54$ & $315(45.9 \%)$ & $659(43.3 \%)$ & \\
\hline $55-79$ & 251 (36.5\%) & 599 (39.4\%) & \\
\hline $80+$ & $3(0.4 \%)$ & $7(0.5 \%)$ & \\
\hline Age at onset of symptoms & $23.6(18.9)$ & $24.5(19.2)$ & 0.321 \\
\hline$<12$ & $230(37.3 \%)$ & $481(35.7 \%)$ & \\
\hline $12-18$ & $57(9.3 \%)$ & $124(9.2 \%)$ & \\
\hline$>18$ & $329(53.4 \%)$ & $742(55.1 \%)$ & \\
\hline Gender & & & 0.964 \\
\hline Female & $428(62.3 \%)$ & $951(62.4 \%)$ & \\
\hline Male & $259(37.7 \%)$ & $573(37.6 \%)$ & \\
\hline Ethnicity & & & 0.024 \\
\hline Caucasian & $514(75.5 \%)$ & $1218(80.6 \%)$ & \\
\hline Non-Caucasian & $167(24.5 \%)$ & $293(19.4 \%)$ & \\
\hline BMI $\left(\mathrm{kg} / \mathrm{m}^{2}\right)$ & $30.8(7.4)$ & $30.9(6.9)$ & 0.747 \\
\hline Smoking status & & & $<0.001$ \\
\hline Never smoked & $428(62.7 \%)$ & $1053(70.8 \%)$ & \\
\hline Ex-smoker & $211(30.9 \%)$ & $403(27.1 \%)$ & \\
\hline Current smoker & $44(6.4 \%)$ & $32(2.2 \%)$ & \\
\hline Atopic disease & $457(66.7 \%)$ & $912(60.9 \%)$ & $<0.001$ \\
\hline Spinal bone density (T-Score) & $-0.7(1.4)$ & $-0.7(1.4)$ & 0.912 \\
\hline $\begin{array}{l}\text { Femoral neck bone density } \\
\text { (T-Score) }\end{array}$ & $-0.5(1.1)$ & $-0.4(1.1)$ & 0.446 \\
\hline $\mathrm{FEV}_{1}(\%$ predicted $)$ & $67.7(22.0)$ & $64.2(20.8)$ & 0.001 \\
\hline FVC (\% predicted) & $83.2(20.0)$ & $83.0(19.5)$ & 0.814 \\
\hline $\mathrm{FEV}_{1} / \mathrm{FVC}$ & $65.0(14.8)$ & $62.7(18.4)$ & 0.007 \\
\hline $\begin{array}{l}\text { Residual volume (\% } \\
\text { predicted) }\end{array}$ & $120.7(43.7)$ & $136.3(43.7)$ & $<0.001$ \\
\hline $\begin{array}{l}\text { Total lung capacity (\% } \\
\text { predicted) }\end{array}$ & $100.5(17.6)$ & $105.0(19.1)$ & 0.007 \\
\hline ACQ6 Score & $3.0(1.4)$ & $2.9(1.4)$ & 0.060 \\
\hline Rescue steroids in last year & $4(2,6)$ & $4(2,7)$ & 0.005 \\
\hline $\begin{array}{l}\text { Hospital admissions for } \\
\text { asthma in last year }\end{array}$ & $0(0,2)$ & $0(0,1)$ & 0.190 \\
\hline Invasive ventilations (ever) & $35(7.2 \%)$ & $158(11.0 \%)$ & $<0.001$ \\
\hline Eczema & $32(4.9 \%)$ & $30(2.0 \%)$ & $<0.001$ \\
\hline Nasal polyps & $80(12.3 \%)$ & $273(18.2 \%)$ & $<0.001$ \\
\hline Gastro-oesophageal reflux & $166(25.4 \%)$ & $198(13.2 \%)$ & $<0.001$ \\
\hline Depression or anxiety & $85(13.0 \%)$ & $93(6.2 \%)$ & $<0.001$ \\
\hline $\begin{array}{l}\text { Blood eosinophil count } \\
\left(\mathrm{N} / 10^{9} \mathrm{~L}\right)\end{array}$ & $0.30(0.13,0.54)$ & $0.36(0.18,0.60)$ & $<0.001$ \\
\hline $\begin{array}{l}\text { Highest blood eosinophil } \\
\text { count }\left(\mathrm{N} / 10^{9} \mathrm{~L}\right) \dagger\end{array}$ & $0.50(0.30,0.82)$ & $0.70(0.40,1.10)$ & $<0.001$ \\
\hline FeNO (ppb) & $36.0(17.0,69.0)$ & $41.0(22.0,76.5)$ & $<0.001$ \\
\hline $\lg E(I U / m L)$ & $182(57,524)$ & $180(60,467)$ & 0.726 \\
\hline Maintenance oral steroids & $230(33.5 \%)$ & $906(59.9 \%)$ & $<0.001$ \\
\hline $\begin{array}{l}\text { Maintenance oral steroid } \\
\text { dose }(\mathrm{mg})\end{array}$ & $6(0,15)$ & $10(8,15)$ & $<0.001$ \\
\hline $\begin{array}{l}\text { Inhaled steroid dose (mcg, } \\
\text { BDP equivalent) }\end{array}$ & $2000(1600,2000)$ & $2000(1600,2000)$ & 0.056 \\
\hline LAMA & 348 (50.9\%) & 806 (54.6\%) & $<0.001$ \\
\hline
\end{tabular}

Continued
Table 3 Continued

\begin{tabular}{lccr}
\hline & $\begin{array}{l}\text { No biologic therapy } \\
(\mathrm{n}=687)\end{array}$ & $\begin{array}{l}\text { Biologic therapy } \\
(\mathrm{n}=1524)\end{array}$ & P value \\
\hline Theophylline & $177(25.9 \%)$ & $414(27.7 \%)$ & 0.015 \\
\hline SABA & $644(94.0 \%)$ & $1434(95.7 \%)$ & 0.007 \\
$\begin{array}{l}\text { Leukotriene receptor } \\
\text { antagonist }\end{array}$ & $378(56.2 \%)$ & $662(45.4 \%)$ & $<0.001$ \\
\hline Maintenance macrolide & $64(9.6 \%)$ & $135(9.3 \%)$ & 0.077 \\
\hline Nebuliser & $154(22.7 \%)$ & $377(25.4 \%)$ & 0.044 \\
\hline Prior anti-lgE therapy & $0(0.0 \%)$ & $251(16.8 \%)$ & $<0.001$ \\
\hline
\end{tabular}

${ }^{*}$ Mean (SD), median (IQR) or count (\%) as appropriate.

tHighest blood count is the highest recorded in available prior medical records.

ACQ, Asthma Control Questionnaire; BMI, body mass index; FeNO, fractional exhaled nitric oxide; LAMA, long-acting antimuscarinic antagonist; SABA, short acting $\beta 2$-agonist.

Compared with anti-IL-5/5R treated patients, anti-IgE treated patients were younger at first assessment (47.6 vs 50.7 years old; $\mathrm{p}<0.001)$, had an earlier onset of symptoms (15.2 vs 27.5 years old; $\mathrm{p}<0.001)$, were more likely to be female $(66.9 \%$ vs $61.0 \% ; \mathrm{p}=0.053)$ and had a higher reported history of atopic disease $(86.7 \%$ vs $52.6 \% ; \mathrm{p}<0.001)$. In contrast, nasal polyposis was more prevalent in the anti-IL5/5R patients $(20.8 \%$ vs $11.1 \%, \mathrm{p}<0.001)$. Prescribing of other asthma medications also differed with prescription of mOCS more prevalent in the anti-IL-5/5R patients $(65.0 \%$ vs $44.6 \%, \mathrm{p}<0.001)$ and the reverse being true for LTRA $(43.3 \%$ vs $52.4 \%, p=0.014)$. In keeping with the specific clinical phenotypes these two classes of biologic therapies are targeted at, significant differences in T2 biomarker levels were observed with a higher registration $(0.40$ vs $0.22 ; \mathrm{p}<0.001)$ and historic (and 0.72 vs 0.50 ; $\mathrm{p}<0.001)$ blood eosinophil count in those on anti-IL5/5R therapies and a higher total IgE in those on anti-IgE treatment (294 vs $143, \mathrm{p}<0.001$ ) (table 4 ). Interestingly although $22.6 \%$ of biologics patients were prescribed omalizumab, $44.8 \%$ of the total biologics cohort met published eligibility criteria for this therapeutic option.

\section{Comparison of T2-high and T2-low patients}

We identified 992 of $2225(44.6 \%)$ patients categorised as T2-high and $210(9.4 \%)$ T2-low using our composite biomarker definitions. Compared with T2-low patients, those in the T2-high group were more likely to be male $(39.4 \%$ vs $32.9 \%, p=0.076)$, have an older age of symptom onset ( 25.9 vs 17.1 years old, $\mathrm{p}<0.001$ ), be a never-smoker $(69.7 \%$ vs $59.1 \%, \mathrm{p}<0.001)$, have nasal polyposis $(19.5 \%$ vs $10.8 \%$, $\mathrm{p}=0.006)$ and have more severe airflow obstruction $\left(\mathrm{FEV}_{1} /\right.$ FVC 63.6 vs $66.7, p=0.007)$. In contrast, T2-low patients had a higher body mass index (BMI) $(32.1$ vs 30.2, p<0.001), higher prevalence of depression and anxiety $(12.3 \%$ vs $7.6 \%$, $\mathrm{p}=0.040)$ and higher rate of current smoking (8.2\% vs $2.2 \%$, $\mathrm{p}<0.001)$. A greater proportion of T2-low patients were treated with $\operatorname{mOCS}(57.9 \%$ vs $42.1 \%, \mathrm{p}<0.001)$ as well as theophylline $(35.1 \%$ vs $23.4 \%, \mathrm{p}=0.001)$ and more likely to have a home nebuliser $(35.1 \%$ vs $19.6 \%, \mathrm{p}<0.001)$. A history of atopic disease was also more prevalent in the T2-low group $(71.5 \%$ vs $61.8 \%, \mathrm{p}=0.028)$ although the median $\mathrm{IgE}$ was higher in the T2-high group (189 vs $155, \mathrm{p}=0.007)$. Importantly, although the blood eosinophil count on registration was used to define T2 status, analysis of the historic blood eosinophil counts highlighted a median count of $0.35(0.13$, 0.60 ) in the T2-low group (table 5). 


\begin{tabular}{|c|c|c|c|}
\hline & Anti-lgE $(n=329)$ & Anti-IL5 ( $n=1120)$ & $P$ value \\
\hline Age at first assessment & $47.6(14.6)$ & $50.7(14.1)$ & $<0.001$ \\
\hline $18-34$ & $71(21.6 \%)$ & $167(14.9 \%)$ & \\
\hline $35-54$ & $140(42.6 \%)$ & $481(43.0 \%)$ & \\
\hline $55-79$ & $117(35.6 \%)$ & $464(41.5 \%)$ & \\
\hline $80+$ & $1(0.3 \%)$ & $6(0.5 \%)$ & \\
\hline Age at onset of symptoms & $15.2(16.0)$ & $27.5(19.2)$ & $<0.001$ \\
\hline$<12$ & $168(60.6 \%)$ & $279(27.8 \%)$ & \\
\hline $12-18$ & $26(9.4 \%)$ & $95(9.5 \%)$ & \\
\hline$>18$ & $83(30.0 \%)$ & $630(62.7 \%)$ & \\
\hline Gender & & & 0.053 \\
\hline Female & $220(66.9 \%)$ & $683(61.0 \%)$ & \\
\hline Male & 109 (33.1\%) & $437(39.0 \%)$ & \\
\hline Ethnicity & & & 0.143 \\
\hline Caucasian & $261(79.3 \%)$ & 895 (80.8\%) & \\
\hline Non-Caucasian & $68(20.7 \%)$ & $213(19.2 \%)$ & \\
\hline BMI $\left(\mathrm{kg} / \mathrm{m}^{2}\right)$ & $31.3(6.8)$ & $30.7(7.0)$ & 0.183 \\
\hline Smoking status & & & 0.757 \\
\hline Never smoked & $233(72.8 \%)$ & $763(69.8 \%)$ & \\
\hline Ex-smoker & $81(25.3 \%)$ & $307(28.1 \%)$ & \\
\hline Current smoker & $6(1.9 \%)$ & $23(2.1 \%)$ & \\
\hline Atopic disease & $281(86.7 \%)$ & $578(52.6 \%)$ & $<0.001$ \\
\hline Spinal bone density (T-Score) & $-0.7(1.4)$ & $-0.6(1.4)$ & 0.593 \\
\hline $\begin{array}{l}\text { Femoral neck bone density } \\
\text { (T-Score) }\end{array}$ & $-0.3(1.1)$ & $-0.5(1.2)$ & 0.062 \\
\hline $\mathrm{FEV}_{1}(\%$ predicted $)$ & $63.4(21.7)$ & $64.9(20.6)$ & 0.321 \\
\hline FVC (\% predicted) & $82.4(18.0)$ & $83.7(20.2)$ & 0.369 \\
\hline $\mathrm{FEV}_{1} / \mathrm{FVC}$ & $61.8(14.7)$ & $63.0(19.8)$ & 0.355 \\
\hline $\begin{array}{l}\text { Residual volume (\% } \\
\text { predicted) }\end{array}$ & $138.7(44.9)$ & $136.8(43.6)$ & 0.684 \\
\hline $\begin{array}{l}\text { Total lung capacity (\% } \\
\text { predicted) }\end{array}$ & $105.6(17.5)$ & $105.4(19.6)$ & 0.913 \\
\hline ACQ6 Score & $2.8(1.5)$ & $2.9(1.4)$ & 0.178 \\
\hline Rescue steroids in last year & $4(2,6)$ & $4(3,7)$ & 0.020 \\
\hline $\begin{array}{l}\text { Hospital admissions for } \\
\text { asthma in last year }\end{array}$ & $0(0,1)$ & $0(0,1)$ & 0.047 \\
\hline Invasive ventilations (ever) & $44(14.1 \%)$ & $108(10.2 \%)$ & 0.134 \\
\hline Eczema & $7(2.2 \%)$ & $17(1.5 \%)$ & 0.748 \\
\hline Nasal polyps & $36(11.1 \%)$ & $230(20.8 \%)$ & $<0.001$ \\
\hline Gastro-oesophageal reflux & $44(13.5 \%)$ & $145(13.1 \%)$ & 0.979 \\
\hline Depression or anxiety & $21(6.5 \%)$ & $65(5.9 \%)$ & 0.926 \\
\hline $\begin{array}{l}\text { Blood eosinophil count } \\
\left(\mathrm{N} / 10^{9} \mathrm{~L}\right)\end{array}$ & $0.22(0.10,0.50)$ & $0.40(0.20,0.68)$ & $<0.001$ \\
\hline $\begin{array}{l}\text { Highest blood eosinophil } \\
\text { count }\left(\mathrm{N} / 10^{9} \mathrm{~L}\right) \dagger\end{array}$ & $0.50(0.30,0.80)$ & $0.72(0.50,1.10)$ & $<0.001$ \\
\hline FeNO (ppb) & $31.0(18.0,61.0)$ & $44.0(24.0,81.0)$ & $<0.001$ \\
\hline $\lg E(I U / m L)$ & $294(151,485)$ & $143(47,407)$ & $<0.001$ \\
\hline Maintenance oral steroids & $145(44.6 \%)$ & $723(65.0 \%)$ & $<0.001$ \\
\hline $\begin{array}{l}\text { Maintenance oral steroid } \\
\text { dose }(\mathrm{mg})\end{array}$ & $10(5,15)$ & $10(8,18)$ & 0.054 \\
\hline $\begin{array}{l}\text { Inhaled steroid dose (mcg, } \\
\text { BDP equivalent) }\end{array}$ & $2000(1600,2000)$ & $2000(1600,2000)$ & 0.029 \\
\hline LAMA & $176(55.0 \%)$ & $589(54.4 \%)$ & 0.859 \\
\hline Theophylline & $102(31.4 \%)$ & $289(26.4 \%)$ & 0.106 \\
\hline
\end{tabular}

Table 4 Continued

\begin{tabular}{lccr}
\hline & Anti-IgE $(\mathrm{n}=329)$ & Anti-IL5 $(\mathrm{n}=1120)$ & P value \\
\hline SABA & $313(96.6 \%)$ & $1054(95.7 \%)$ & 0.764 \\
$\begin{array}{l}\text { Leukotriene receptor } \\
\text { antagonist }\end{array}$ & $164(52.4 \%)$ & $466(43.3 \%)$ & 0.014 \\
Maintenance macrolide & $28(9.0 \%)$ & $103(9.6 \%)$ & 0.713 \\
Nebuliser & $89(27.9 \%)$ & $267(24.5 \%)$ & 0.442 \\
\hline Prior anti-IgE therapy & $140(42.8 \%)$ & $77(7.0 \%)$ & $<0.001$ \\
\hline
\end{tabular}

${ }^{*}$ Mean (SD), median (IQR) or count (\%) as appropriate.

tHighest blood count is the highest recorded in available prior medical records. ACQ, Asthma Control Questionnaire; BMI, body mass index; FeNO, fractional exhaled nitric oxide; LAMA, long-acting antimuscarinic antagonist; SABA, short acting $\beta 2$-agonist.

\section{DISCUSSION}

The UKSAR represents the largest national registry of its kind in the world recruiting patients with severe asthma who have been systematically evaluated at specialist asthma centres within the UK. This includes assessment and confirmation of severity, inflammatory phenotype, therapeutic intervention and related comorbidities. We report that the majority have evidence of T2 inflammation despite high rates of systemic corticosteroid use, that T2 low patients have a higher BMI and prevalence of anxiety/depression and that a significant unmet need exists despite currently available therapies.

In line with the increasing availability of biologic therapies targeting the T2-inflammatory pathway, we report a high uptake of biologic therapies in UKSAR, reflecting the fact that referrals and registry enrolment are prioritised by centres for biologic patients. This is despite eligibility criteria that are considered among the most demanding in the world. ${ }^{20-23}$ However, we also highlight the sobering finding that over half of the UKSAR are on maintenance OCS, continue to have a high exacerbation rate averaging four acute OCS courses/ year and remain poorly controlled with an average ACQ6 of 2.9 at assessment.

The relative proportions of each biologic prescribed in the UKSAR reflect the duration of availability of the specific therapy at the time of this analysis, the size of the eligible population as well as individual prescribing habits of physicians. However, it is interesting to note that despite the relatively recent arrival of anti-IL5/5R therapies, these make up over $75 \%$ of all biologic prescribing. This may in part to relate to the relatively high use of mOCS to manage severe asthma in the UK and the lack of controlled data supporting OCS sparing efficacy with omalizumab compared with mepolizumab ${ }^{24}$ or benralizumab but may also relate to the prescribing limitations of body weight and IgE levels which limit access to omalizumab. ${ }^{25}$

In addition, many of the clinical characteristics that differentiate the patients prescribed anti-IgE from anti-IL5/5R in UKSAR are in keeping with current understanding of allergic and nonallergic asthma phenotypes as well as the results of responder analyses conducted following the phase 3 trials of T2 biologics. ${ }^{26}$ Specifically, we report that younger atopic patients with an earlier disease onset were proportionately more likely to be prescribed omalizumab compared with the adult-onset older patients with comorbid nasal polyposis who were in much higher numbers in the anti-IL-5/5R group.

Our definition of T2-low severe asthma uses cut-points for blood eosinophil count and $\mathrm{FeNO}$ which have been identified in phase 3 clinical trials of biologics targeting anti-IL5/5R and anti-IL4R- $\alpha$ respectively, and which have identified little benefit of these therapies when the blood eosinophil count is $<150$ cells/ 


\begin{tabular}{|c|c|c|c|}
\hline & T2-low $(n=210)$ & T2-high ( $n=992)$ & $P$ value \\
\hline Age at first assessment & $48.1(15.1)$ & $48.8(14.3)$ & 0.522 \\
\hline $18-34$ & $47(22.4 \%)$ & $173(17.5 \%)$ & \\
\hline $35-54$ & $84(40.0 \%)$ & $456(46.1 \%)$ & \\
\hline $55-79$ & $78(37.1 \%)$ & $360(36.4 \%)$ & \\
\hline $80+$ & $1(0.5 \%)$ & $1(0.1 \%)$ & \\
\hline Age at onset of symptoms & $17.1(16.6)$ & $25.9(19.1)$ & $<0.001$ \\
\hline$<12$ & $96(53.6 \%)$ & $282(31.0 \%)$ & \\
\hline $12-18$ & $18(10.1 \%)$ & $88(9.7 \%)$ & \\
\hline$>18$ & $65(36.3 \%)$ & $540(59.3 \%)$ & \\
\hline Gender & & & 0.076 \\
\hline Female & $141(67.1 \%)$ & $601(60.6 \%)$ & \\
\hline Male & $69(32.9 \%)$ & $391(39.4 \%)$ & \\
\hline Ethnicity & & & 0.012 \\
\hline Caucasian & $178(85.2 \%)$ & $748(76.4 \%)$ & \\
\hline Non-Caucasian & $31(14.8 \%)$ & $231(23.6 \%)$ & \\
\hline BMI $\left(\mathrm{kg} / \mathrm{m}^{2}\right)$ & $32.1(7.8)$ & $30.2(6.7)$ & $<0.001$ \\
\hline Smoking status & & & $<0.001$ \\
\hline Never smoked & $123(59.1 \%)$ & $688(69.7 \%)$ & \\
\hline Ex-smoker & $68(32.7 \%)$ & $277(28.1 \%)$ & \\
\hline Current smoker & $17(8.2 \%)$ & $22(2.2 \%)$ & \\
\hline Atopic disease & $148(71.5 \%)$ & $607(61.8 \%)$ & 0.028 \\
\hline $\begin{array}{l}\text { Spinal bone density (T- } \\
\text { Score) }\end{array}$ & $-1.0(1.4)$ & $-0.7(1.4)$ & 0.010 \\
\hline $\begin{array}{l}\text { Femoral neck bone density } \\
\text { (T-Score) }\end{array}$ & $-0.5(1.1)$ & $-0.4(1.1)$ & 0.314 \\
\hline $\mathrm{FEV}_{1}(\%$ predicted $)$ & $66.2(22.6)$ & $66.5(20.6)$ & 0.858 \\
\hline FVC (\% predicted) & $80.6(19.8)$ & $84.4(18.7)$ & 0.020 \\
\hline $\mathrm{FEV}_{1} / \mathrm{FVC}$ & $66.7(14.8)$ & $63.6(13.7)$ & 0.007 \\
\hline $\begin{array}{l}\text { Residual volume (\% } \\
\text { predicted) }\end{array}$ & $122.8(44.1)$ & $128.1(44.7)$ & 0.450 \\
\hline $\begin{array}{l}\text { Total lung capacity (\% } \\
\text { predicted) }\end{array}$ & $96.2(22.8)$ & $103.3(17.7)$ & 0.016 \\
\hline ACQ6 Score & $3.1(1.3)$ & $2.9(1.4)$ & 0.161 \\
\hline Rescue steroids in last year & $4(1,7)$ & $4(2,8)$ & 0.014 \\
\hline $\begin{array}{l}\text { Hospital admissions for } \\
\text { asthma in last year }\end{array}$ & $0(0,2)$ & $0(0,1)$ & 0.005 \\
\hline Invasive ventilations (ever) & $26(15.5 \%)$ & $73(8.4 \%)$ & $<0.001$ \\
\hline Eczema & $8(3.9 \%)$ & $29(3.0 \%)$ & 0.341 \\
\hline Nasal polyps & $22(10.8 \%)$ & $190(19.5 \%)$ & 0.006 \\
\hline Gastro-oesophageal reflux & $41(20.2 \%)$ & $173(17.8 \%)$ & 0.317 \\
\hline Depression or anxiety & $25(12.3 \%)$ & $74(7.6 \%)$ & 0.040 \\
\hline $\begin{array}{l}\text { Blood eosinophil count } \\
\left(\mathrm{N} / 10^{9} \mathrm{~L}\right)\end{array}$ & $0.07(0.01,0.10)$ & $0.50(0.30,0.70)$ & $<0.001$ \\
\hline $\begin{array}{l}\text { Highest blood eosinophil } \\
\text { count }\left(\mathrm{N} / 10^{9} \mathrm{~L}\right)^{\dagger}\end{array}$ & $0.35(0.13,0.60)$ & $0.76(0.50,1.15)$ & $<0.001$ \\
\hline FeNO (ppb) & $14.0(9.0,18.0)$ & $60.0(39.0,94.0)$ & $<0.001$ \\
\hline $\lg E(I U / m L)$ & $155(34,437)$ & $189(67,509)$ & 0.007 \\
\hline Maintenance oral steroids & $121(57.9 \%)$ & $415(42.1 \%)$ & $<0.001$ \\
\hline $\begin{array}{l}\text { Maintenance oral steroid } \\
\text { dose }(\mathrm{mg})\end{array}$ & $10(5,20)$ & $10(5,15)$ & 0.013 \\
\hline
\end{tabular}

Continued
Table 5 Continued

\begin{tabular}{lccc}
\hline & T2-low $(\mathrm{n}=210)$ & T2-high $(\mathrm{n}=992)$ & P value \\
\hline $\begin{array}{l}\text { Inhaled steroid dose }(\mathrm{mcg}, \\
\text { BDP equivalent) }\end{array}$ & $2000(1600,2000)$ & $2000(1600,2000)$ & 0.733 \\
LAMA & $116(56.0 \%)$ & $527(54.2 \%)$ & 0.790 \\
Theophylline & $73(35.1 \%)$ & $228(23.4 \%)$ & 0.001 \\
\hline SABA & $196(94.2 \%)$ & $937(95.3 \%)$ & 0.801 \\
$\begin{array}{l}\text { Leukotriene receptor } \\
\text { antagonist }\end{array}$ & $107(53.0 \%)$ & $488(50.8 \%)$ & 0.784 \\
Maintenance macrolide & $23(11.4 \%)$ & $76(7.9 \%)$ & 0.248 \\
Nebuliser & $73(35.1 \%)$ & $189(19.6 \%)$ & $<0.001$ \\
\hline Prior anti-lgE therapy & $36(17.3 \%)$ & $99(10.2 \%)$ & 0.008 \\
\hline
\end{tabular}

*Mean (SD), median (IQR) or count (\%) as appropriate.

tHighest blood count is the highest recorded in available prior medical records. ACQ, Asthma Control Questionnaire; BMI, body mass index; FeNO, fractional exhaled nitric oxide; LAMA, long-acting antimuscarinic antagonist; SABA, short acting $\beta 2$-agonist.

$\mu \mathrm{L}$ or FeNO is $<25 \mathrm{ppb}^{16}{ }^{17}$ Although previous reports of mildmoderate asthma using sputum analysis have described an approximate 50-50 split between T2-high and T2-low phenotypes, ${ }^{2728}$ it is increasingly recognised that severe asthma is predominantly associated with a T2-high phenotype. In keeping with this, we found that while the median blood eosinophil count at registration in the UKSAR was 0.3 cells $\times 10^{9} / \mathrm{L}$, the previous historic (prior to UKSAR registration) median level was 0.62 cells $\times 10^{9} / \mathrm{L}$ with levels greater than 0.4 cells $\times 10^{9} / \mathrm{L}$ in $75 \%$ of the cohort, reflecting prominent blood eosinophilia despite substantial background treatment. The median FeNO of $39 \mathrm{ppb}$ further supports background T2 inflammatory pathway activation in this group. Recent data have demonstrated that when corticosteroids are down-titrated in a UK severe asthma population, T2-biomarkers increase, with the maximal prevalence of T2-low severe asthma reported at $5 \%{ }^{29}$

Indeed, what is perhaps surprising is that such high T2 biomarker levels were evident despite the very high inhaled and systemic steroid utilisation in this cohort and despite adherence assessment. Dividing our cohort into T2-high and T2-low groups based on the combination of blood eosinophils and $\mathrm{FeNO}$ at registration highlighted that only a minority of patients fulfilled the biomarker definition of T2-low asthma. Moreover, analysis of the historic blood eosinophil count in the T2-low group revealed a median level consistent with a T2-high diagnosis and more than $25 \%$ of patients had readings in excess 0.6 cells $\times 10^{9} / \mathrm{L}$. Taken together it highlights the difficulties in labelling asthmatics as T2-low given the variability of biomarkers such as the blood eosinophil count and $\mathrm{FeNO}$ in relation to background corticosteroid treatment, ${ }^{29}{ }^{30}$ which are known to suppress these biomarkers. Consequently, this and any comparison of T2 phenotypes using biomarker stratification at a single timepoint, when on high-dose corticosteroid treatment is challenging and prone to misclassification of patients. It also suggests that corticosteroid treatment in these persistently symptomatic patients may be elevated beyond a point where there would be any additional therapeutic benefit. Consistent with this, we noted that $58 \%$ of these patients were on maintenance OCS.

In light of the high level of morbidity and mortality which we have previously highlighted to be associated with systemic steroids $^{31}$ and the recognition that this therapy is only associated with clinical benefit in the presence of $\mathrm{T} 2$ inflammation, it would 
suggest a greater level of OCS stewardship is required in these patients. In addition, further work is required to establish the mechanism of persistent poor symptom control in these patients who lack objective evidence of T2 inflammation as this presumably drives some clinicians to increase corticosteroid treatment despite the absence of T2 inflammation. Importantly this will require detailed consideration of extrapulmonary factors given the elevated BMI and reduced total lung capacity observed in this group. ${ }^{32}$ It is also striking that the rescue corticosteroid use in this T2-biomarker low group is identical to the T2-high group, given T2-biomarkers have consistently been shown to have prognostic value in terms of exacerbation risk. Additionally, one would anticipate therapeutic benefit from corticosteroids in exacerbations in the T2-high population, but it remains unclear if this is also the case in biomarker low patients. As such, understanding the mechanism and inflammatory phenotype of exacerbation events in T2-biomarker low patients is an important future research question. This same issue applies to the residual $50 \%$ of exacerbation events seen in clinical trials of biologic therapies targeting T2-pathways and clinical trials are underway to try and explore this issue further in patients on mepolizumab and benralizumab (NCT03324230 and NCT04102800).

It was also noteworthy that we did not see higher prescription rates of LAMA and/or macrolide therapy in the T2 low group despite these therapies frequently being discussed as possible therapeutic options when a T2 inflammatory signal appears absent in severe asthma and particularly given the high symptom burden in this population. We cannot identify if these therapies were previously tried and withdrawn but ongoing follow-up will identify any additional treatment in these patients and if corticosteroid treatment is reduced.

Several observations require further investigation. One of these is the differences observed in biologic prescribing between Caucasian and non-Caucasian patients in the UK. While it is possible that variances in access to care, cultural and language barriers as well as possible underlying endotype differences may all play a role this area, and we have noted different disease by ethnicity in $\mathrm{UKSAR}^{33}$ which we are actively exploring further in primary care datasets. Additionally, the higher rate of depression and anxiety seen in the T2-low group as well as the larger group of patients not prescribed a biologic therapy deserves attention. These patients are frequently on mOCS which have well-recognised psychological effects ${ }^{84}$ but our data suggests it is not due to a higher rate of exacerbations, hospitalisation or a poorer level of asthma control in the non-biologic group. The impact of depression and anxiety, and overall quality of life, in severe asthma is a major issue that needs further consideration.

The size of our registry cohort now gives us the power to understand real-world outcomes of severe asthma patients across a common multidisciplinary healthcare system. Annual clinical reviews are being entered as registry follow-up entries and will allow us to better understand the influence of baseline differences on disease trajectory. A further important utility of the registry is its potential to highlight the characteristics of UK severe asthma to inform the commissioning process and also to understand where there might be variation in care between centres, and why this might be the case. To facilitate this, we have recently reviewed the registry data-metrics, in particular reflecting on our current results and in partnership with the NHS England Quality Improvement initiative, and have revised the data-fields in UKSAR for 2020 onwards (online supplemental appendix 2 and 3 ).

In summary, the UKSAR describes the characteristics of a very large cohort of severe asthmatics in routine clinical care across the UK with over 1500 patients treated with biologic therapies. It highlights current prescribing patterns, the predominance of the T2-high clinical phenotype in severe asthma and offers the prospect of providing novel insights across a range of research areas including real world responses to biologic therapies and the natural history of severe asthma.

\section{Author affiliations}

${ }^{1}$ Guy's Severe Asthma Centre, Guy's and St Thomas' NHS Foundation Trust, UK ${ }^{2}$ Asthma UK Centre, King's College London, UK

${ }^{3}$ Centre for Public Health, Queen's University Belfast School of Medicine, Dentistry and Biomedical Sciences, UK

${ }^{4}$ Respiratory Medicine, Barts Health NHS Trust, London, UK

${ }^{5}$ Lung Division, Royal Brompton and Harefield NHS Foundation Trust, London, UK

${ }^{6}$ Respiratory Medicine, Portsmouth Hospitals NHS Trust, Portsmouth, UK

${ }^{7}$ Respiratory Medicine, Cambridge University Hospitals Trust, Cambridge, UK

${ }^{8}$ Respiratory Medicine, Russells Hall Hospital, Dudley, UK

${ }^{9}$ Heartlands Hospital, Heart of England NHS Foundation Trust, Birmingham, UK

${ }^{10}$ University of Birmingham, UK

${ }^{11}$ Respiratory Medicine, Gloucestershore Royal Hospital, Gluocester, UK

${ }^{12}$ Wythenshawe Hospital, Manchester NHS Foundation Trust, UK

${ }^{13}$ Respiratory Medicine, University Plymouth NHS Trust, Plymouth, UK

${ }^{14}$ Centre for Experimental Medicine, Queen's University Belfast School of Medicine,

Dentistry and Biomedical Sciences, UK

${ }^{15}$ Belfast Health \& Social Care NHS Trust, UK

Acknowledgements We thank the data input and medical staff in the UK Difficult Asthma Centres.

Collaborators Dr Paul Dilworth, Royal Free London NHS Trust; Dr Aashish Vyas, Lancashire Teaching Hospital; Dr Rekha Chadhuri, Gartnavel General Hospital; Dr Deepak Subramnian, University Hospitals of Derby \& Bunton NHS; Dr Paddy Dennison, Southampton General Hospital.

Contributors CJJ, JB, PEP, AMG, TB, RG, MD, AM, SM, RN, MP and LGH made substantial contributions to data acquisition, data analysis and interpretation, and drafting the manuscript. The final manuscript was approved by all the authors prior to submission.

Funding The authors have not declared a specific grant for this research from any funding agency in the public, commercial or not-for-profit sectors.

Patient consent for publication Not required.

Ethics approval Approval for collection and analysis of pseudonymised UKSAR data was granted by ORECNI (15/NI/0196).

Provenance and peer review Not commissioned; externally peer reviewed.

Data availability statement No data are available.

Open access This is an open access article distributed in accordance with the Creative Commons Attribution Non Commercial (CC BY-NC 4.0) license, which permits others to distribute, remix, adapt, build upon this work non-commercially, and license their derivative works on different terms, provided the original work is properly cited, appropriate credit is given, any changes made indicated, and the use is non-commercial. See: http://creativecommons.org/licenses/by-nc/4.0/.

\section{ORCID iDs}

Paul E Pfeffer http://orcid.org/0000-0003-0369-2885

Adel H Mansur http://orcid.org/0000-0002-8615-8778

\section{REFERENCES}

1 Chung KF. Sally Wenzel for the European respiratory Society/American thoracic Society severe asthma international guidelines Task force. European Respiratory Journa/2014;44:1378-137.

2 von Bülow A, Kriegbaum M, Backer V, et al. The prevalence of severe asthma and low asthma control among Danish adults. J Allergy Clin Immunol Pract 2014;2:759-67.

3 Hekking P-PW, Wener RR, Amelink M, et al. The prevalence of severe refractory asthma. J Allergy Clin Immunol 2015;135:896-902.

4 Heaney LG, Brightling CE, Menzies-Gow A, et al. British thoracic Society difficult asthma network. Refractory asthma in the UK: cross-sectional findings from a UK multicentre registry, Thorax 2010;65:787-94.

5 Burn J, Sims AJ, Patrick H, et al. Efficacy and safety of bronchial thermoplasty in clinical practice: a prospective, longitudinal, cohort study using evidence from the UK severe asthma registry. BMJ Open 2019;9:e026742.

6 Burn J, Sims AJ, Keltie K, et al. Procedural and short-term safety of bronchial thermoplasty in clinical practice: evidence from a national registry and hospital episode statistics. J Asthma 2017;54:872-9. 
7 Chaudhuri R, McSharry C, Heaney LG, et al. Effects of older age and age of asthma onset on clinical and inflammatory variables in severe refractory asthma. Respir Med 2016;118:46-52.

8 Sweeney J, Patterson CC, Menzies-Gow A, et al. Comorbidity in severe asthma requiring systemic corticosteroid therapy: cross-sectional data from the optimum patient care research database and the British thoracic difficult asthma registry. Thorax 2016;71:339-46

9 Gibeon D, Heaney LG, Brightling CE, et al. British thoracic Society difficult asthma network. dedicated severe asthma services improve health-care use and quality of life. Chest 2015;148:870-6.

10 Newby C, Heaney LG, Menzies-Gow A, et al. British thoracic Society severe refractory asthma network. statistical cluster analysis of the British thoracic Society severe refractory asthma registry: clinical outcomes and phenotype stability. PLoS One 2014;9:e102987.

11 O'Neill S, Sweeney J, Patterson CC, et al. British thoracic Society difficult asthma Network. The cost of treating severe refractory asthma in the UK: an economic analysis from the British thoracic Society difficult asthma registry. Thorax 2015;70:376-8.

12 Thomson NC, Chaudhuri R, Heaney LG, et al. Clinical outcomes and inflammatory biomarkers in current smokers and exsmokers with severe asthma. J Allergy Clin Immunol 2013;131:1008-16.

13 Gibeon D, Batuwita K, Osmond M, et al. Obesity-Associated severe asthma represents a distinct clinical phenotype: analysis of the British thoracic Society difficult asthma registry patient cohort according to BMI. Chest 2013;143:406-14.

14 Sweeney J, CEss B, Menzies-Gow A, et al. British thoracic Society difficult asthma network. clinical management and outcome of refractory asthma in the UK from the British thoracic Society difficult asthma registry. Thorax 2012;67:754-6.

15 Bulathsinhala L, Eleangovan N, Heaney LG, et al. Development of the International severe asthma registry (ISAR): a modified Delphi study. J Allergy Clin Immunol Pract 2019;7:578-88.

16 Ortega HG, Yancey SW, Mayer B, et al. Severe eosinophilic asthma treated with mepolizumab stratified by baseline eosinophil thresholds: a secondary analysis of the DREAM and MENSA studies. Lancet Respir Med 2016:4:549-56.

17 Castro M, Corren J, Pavord ID, et al. Dupilumab efficacy and safety in moderate-tosevere uncontrolled asthma. N Engl J Med 2018;378:2486-96.

18 Heaney LG, Conway E, Kelly C, et al. Predictors of therapy resistant asthma: outcome of a systematic evaluation protocol. Thorax 2003;58:561-6.

19 Robinson DS, Campbell DA, Durham SR, et al. Asthma and allergy Research group of the National heart and lung Institute. systematic assessment of difficult-to-treat asthma. Eur Respir J 2003:22:478-83.
20 NICE. Omalizumab for treating severe persistent allergic asthma, 2013. Available: https://www.nice.org.uk/guidance/ta278

21 NICE. Mepolizumab for treating severe refractory eosinophilic asthma, 2017. Available: https://www.nice.org.uk/guidance/ta431

22 NICE. Reslizumab for treating severe eosinophilic asthma, 2017. Available: https:// www.nice.org.uk/guidance/TA479

23 NICE. Benralizumab for treating severe eosinophilic asthma, 2019. Available: https:// www.nice.org.uk/guidance/ta565

24 Bel EH, Wenzel SE, Thompson PJ, et al. Oral glucocorticoid-sparing effect of mepolizumab in eosinophilic asthma. N Engl J Med 2014;371:1189-97.

25 Nair P, Wenzel S, Rabe KF, et al. Oral glucocorticoid-sparing effect of Benralizumab in severe asthma. N Engl J Med 2017;376:2448-58.

26 Bleecker ER, Wechsler ME, FitzGerald JM, et al. Baseline patient factors impact on the clinical efficacy of benralizumab for severe asthma. Eur Respir J 2018;52. doi:10.1183/13993003.00936-2018. [Epub ahead of print: 1810 2018].

27 Woodruff PG, Modrek B, Choy DF, et al. T-Helper type 2-driven inflammation defines major subphenotypes of asthma. Am J Respir Crit Care Med 2009;180:388-95.

28 McGrath KW, Icitovic N, Boushey HA, et al. Asthma clinical research network of the National heart, lung, and blood Institute. A large subgroup of mild-to-moderate asthma is persistently non-eosinophilic. Am J Resp Crit Care Med 2012;185:612-9.

29 Heaney LG, Busby J, et al, on behalf of the investigators for the MRC Refractory Asthma Stratification Program. Randomised trial of treatment optimisation in patients with severe asthma using composite type-2 biomarkers to adjust corticosteroid dose versus a symptom/risk-based algorithm. Lancet Respiratory Medicine2020

30 Busby J, Holweg CTJ, Chai A, et al. Change in type-2 biomarkers and related cytokines with prednisolone in uncontrolled severe oral corticosteroid dependent asthmatics: an interventional open-label study. Thorax 2019;74:806-9.

31 Sweeney J, Patterson CC, Menzies-Gow A, et al. Comorbidity in severe asthma requiring systemic corticosteroid therapy: cross-sectional data from the optimum patient care research database and the British thoracic difficult asthma registry. Thorax 2016;71:339-46.

32 McDowell PJ, Heaney LG. Different endotypes and phenotypes drive the heterogeneity in severe asthma. Allergy 2020;75:302-10.

33 Busby J, Jackson DJ, Mansur AH, et al. British thoracic Society winter meeting 2019. Programme and Abstracts; 74:P152.

34 Bloechliger M, Reinau D, Spoendlin J, et al. Adverse events profile of oral corticosteroids among asthma patients in the UK: cohort study with a nested casecontrol analysis. Respir Res 2018;19:75. 